\title{
The Early Diagnostic and Prognostic Value of BIRC5 in Clear-Cell Renal Cell Carcinoma Based on the Cancer Genome Atlas Data
}

\author{
Jingyuan Wang ${ }^{a}$ Min Chen ${ }^{b}$ Chengxue Dang ${ }^{b}$ Hao Zhang ${ }^{b}$ Xin Wang ${ }^{b}$ \\ Jianhao Yin ${ }^{b}$ Rui Jia ${ }^{b}$ Yong Zhang ${ }^{b}$ \\ ${ }^{a}$ Department of Clinical Lab Diagnosis, The First Affiliated Hospital of Xi'an Jiaotong University, Xi'an, China; \\ bepartment of Surgical Oncology, The First Affiliated Hospital of Xi'an Jiaotong University, Xi'an, China
}

\section{Keywords}

Clear-cell renal cell carcinoma - Baculoviral inhibitor of apoptosis repeat containing 5 - The Cancer Genome Atlas database · Early diagnosis · Prognosis

\begin{abstract}
Purpose: The aim of this study was to investigate the role of BIRC5 for early diagnosis and prognosis in clear-cell renal cell carcinoma (ccRCC) by studying the expression of BIRC5 and the correlation between BIRC5 expression and clinicopathological parameters and prognosis in cCRCC. Methods: The BIRC5 expression in ccRCC tissues and normal kidney tissues was measured using the Cancer Genome Atlas database and the Human Protein Atlas database. The correlation between BIRC5 expression and clinicopathological parameters and prognosis in cCRCC was analyzed using UALCAN, the KaplanMeier plotter, GEPIA, and SurvExpress. Thirteen-paired CCRCC plasma samples were used to verify the BIRC5 early diagnosis value of ccRCC. Results: The BIRC5 expression is significantly higher in ccRCC than in normal kidney tissues, and is correlated with the clinical stage and pathological grade of $\operatorname{ccRCC}(p<0.05)$. The result of analyzing the relationship between BIRC5 expression and outcomes in ccRCC indicates that a high BIRC5 expression is an independent prognostic factor affecting the overall survival and disease-free survival of $\operatorname{ccRCC}(p<0.05)$. Compared with normal kidney
\end{abstract}

tissues, the immunohistochemical test shows that BIRC5 is significantly upregulated in CcRCC tissues. mRNA expression levels of BIRC 5 were significantly higher in the cCRCC plasma than normal $(p<0.05)$. Conclusions: The high expression of BIRC5 is an important indicator for the prognosis of $c C R C C$, which makes BIRC5 an effective biomarker for predicting the prognosis of patients in CCRCC. BIRC5 may be a great potential biomarker for early diagnosis of cCRCC.

(c) 2021 The Author(s).

Published by S. Karger AG, Basel

\section{Introduction}

Renal cell carcinoma (RCC) is the third most frequent urological malignant neoplasms worldwide, among which, clear-cell RCC (ccRCC) accounts for approximately $80-90 \%$ [1], being responsible for the most common type of renal malignancy. Because of its high rates of local invasion, metastasis, and acquired chemoresistance, ccRCC is the most lethal RCC histological subtype [2]. If diagnosed at an early stage, ccRCC can be cured by surgery. However, the 5-year disease-specific survival rate of ccRCC is only $12 \%$. What's more, due to the asymptomatic nature of the early ones, $16 \%$ of patients with ccRCC

Jingyuan Wang and Min Chen contributed equally. karger@karger.com www.karger.com/uin

Karger $\stackrel{\text { ' }}{5}$

BOPEN ACCESS
(C) 2021 The Author(s)

Published by S. Karger AG, Basel

This is an Open Access article licensed under the Creative Commons Attribution-NonCommercial-4.0 International License (CC BY-NC) (http://www.karger.com/Services/OpenAccessLicense), applicable to the online version of the article only. Usage and distribution for commercial purposes requires written permission.
Correspondence to:

Yong Zhang, yongzhang761@ mail.xjtu.edu.cn 
are identified with lymph node metastasis or distant metastasis at first diagnosis, and $20-30 \%$ of localized ccRCC patients experience recurrence or metastasis even following surgery, and no effective therapies are found to reduce the risk of recurrence, progression, or death $[3,4]$. Thus, it is important to find reliable biomarkers to facilitate early diagnosis and screen out disease progression of ccRCC.

BIRC5 (also known as survivin) is a cancer-associated protein that inhibits cell death, which is a key member of the inhibitor of apoptosis protein family, encoded by the BIRC5 (baculoviral inhibitor of apoptosis repeat containing 5) gene. Biological function of BIRC5 is involved in the regulation of both apoptosis and cell division when BIRC5 is usually highly expressed in several cancers but not expressed in normal differentiated tissues. BIRC5 expression allows tumor cells to overcome apoptotic checkpoints, while several antitumor agents function through apoptosis activation, BIRC5 expression may contribute to the resistance to anticancer agents $[5,6]$. Increased BIRC5 expression is associated with negative outcomes or survival in various cancers, such as breast, lung, colorectal, prostate, and ovarian cancers [7]. All of these features make BIRC5 a famous molecule among cancer research; however, very few researches have been investigated regarding BIRC5 expression and ccRCC.

The Cancer Genome Atlas (TCGA), having huge data resources of cancers, plays an irreplaceable role in cancer research when providing an opportunity to analyze the associations of various clinicopathologic factors with tumor initiation and progression to cancer researchers [8]. Various computational tools have been developed to aid researchers in carrying out specific TCGA data analyses, and facilitate the study of gene expression variations and survival associations across tumors. In our study, we aimed to examine BIRC5 expression and identify BIRC5 of the prognostic value in the ccRCC using developed computational tools of TCGA; we try to explore the possible mechanisms behind ccRCC development and reveal reliable early diagnosis and prognostic biomarkers and therapeutic targets for ccRCC.

\section{Materials and Methods}

\section{Relationship between BIRC5 Expression and}

Clinicopathological Features

UALCAN (http://ualcan.path.uab.edu) is an easy to use and interactive web portal to perform in-depth analyses of TCGA gene expression data. The gene expression and clinical patient data were downloaded from TCGA, and the analysis results are represented by box plots, KM plots, and heatmaps. Through its links, queried gene expression analysis and chose cancer-type survival analysis results can be acquired [9]. We used UALCAN to analyze the relative expression of BIRC5 across tumor and normal samples, also in various tumor subgroups based on individual cancer stages, tumor grade, and nodal metastasis.

\section{Relationship between BIRC5 Expression and Outcome of} ccRCC

The Kaplan-Meier plotter (http://kmplot.com/analysis/) is capable to assess the effect of $54 \mathrm{~K}$ genes on survival in 21 cancer types (breast, ovarian, lung, gastric cancers; ccRCC and so on). Its gene expression data and relapse-free and overall survival (OS) information are downloaded from GEO, EGA, and TCGA. The patient samples are split into 2 groups according to various quantile expressions of the proposed biomarker. The 2 patient cohorts are compared by a Kaplan-Meier survival plot, and the hazard ratio with $95 \%$ confidence intervals and the $\log$-rank $p$ value is calculated. We used the Kaplan-Meier plotter to analyze the association between BIRC5 expression and OS of ccRCC.

GEPIA (http://gepia.cancer-pku.cn/index.html) is a web server for analyzing the RNA sequencing expression data of 9,736 tumors and 8,587 normal samples from the TCGA and the GTEx projects. It provides multiple customizable functions such as tumor/normal differential expression analysis, patient survival analysis, and similar gene detection [10]. We used GEPIA to analyze the association between BIRC5 expression and disease-free survival (DFS) of ccRCC.

SurvExpress (http://bioinformatica.mty.itesm.mx/SurvExpress) is a comprehensive gene expression database and a webbased tool, providing survival analysis and risk assessment in cancer datasets. It can facilitate performance comparisons of the Kaplan-Meier plotter, PrognoScan, or others, and validations of prognostic and predictive biomarkers for cancer outcomes [11]. To further validate the association between BIRC5 expression and outcome of ccRCC, we used SurvExpress to analyze 468 ccRCC data from TCGA, using COX regression analysis.

Protein Expression of BIRC5 between Tumor Tissues of ccRCC and Normal Kidney Tissues

The Human Protein Atlas (HPA) database (https://www.proteinatlas.org) is capable of detecting $84 \%$ of the human proteome through antibody-based proteomics, and can be used to study gene expression between the tissue of normal and tumors. We used the HPA database to test the immunohistochemistry of BIRC5 between the tissue of normal and ccRCC.

\section{Quantitative Reverse Transcriptase-Polymerase Chain}

\section{Reaction}

In order to explore the differential expression of BIRC5 in the plasma between early ccRCC and normal people, we went forward to validate the BIRC5 expression characteristic in 13 paired ccRCC samples by quantitative reverse transcriptase-polymerase chain reaction. Total RNA was extracted by using the Trizol reagent (Invitrogen, Carlsbad, CA, USA), which was used to generate cDNA by using the SuperScript III RT/Invitrogen with an oligo (dT) primer. Q-PCR was performed using Platinum SYBR Green qPCR SuperMix (Invitrogen) as recommended by the manufacturer. The primers used were as follows: BIRC5, $5^{\prime}$-CGACCCCATAGAGGAACATAAA-3' ${ }^{\prime}$ and $5^{\prime}$-GGAATAAACCCTGGAAGTGGTG-3' 
Fig. 1. The expression of BIRC5 in ccRCC based on TCGA samples $(p<0.05)$. TCGA, the Cancer Genome Atlas; ccRCC, clearcell renal cell carcinoma.

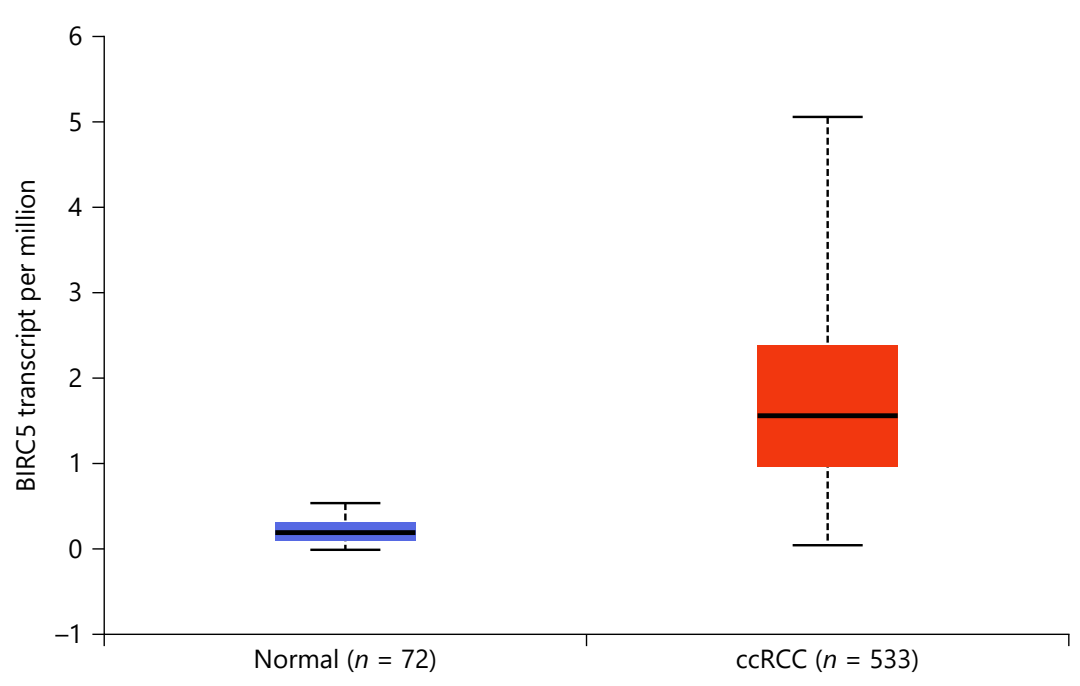

BIRC5 can be used as an independent prognostic predictor for ccRCC patients. Analyzing the association between BIRC5 expression and OS using the Kaplan-Meier plotter, we found the higher BIRC5 expression was significantly related to patient's OS $(p<0.05)$ (Fig. 3a). Then, analyzing the association between BIRC5 expression and DFS using GEPIA, we found the higher BIRC5 expression was significantly related to patient's DFS $(p<0.05)$ (Fig. 3b). Also, to further verify the role of BIRC5 in ccRCC, we used SurvExpress to analyze the data of 468 ccRCC from TCGA using COX regression analysis. The prognostic index was calculated with regard to the BIRC5 mRNA expression level as a dichotomous risk factor. We found that in high-risk ccRCC patients, the expression level of BIRC5 is significantly high (Fig. 3d), and a high expression of BIRC5 is an independent poor prognostic indicator of ccRCC (Fig. 3c).

\section{Protein Expression of BIRC5 Is Higher in Tumor}

Tissues of ccRCC

Analyzing immunohistochemical results of BIRC5 protein in the normal and ccRCC kidney tissue using HPA, we found that antibody for BIRC5 protein is "not detected," the "low"-level in normal tissue, when is "medium" - the "high"-level in ccRCC (Fig. 4). This further confirms that the expression of BIRC5 in ccRCC is higher than in the normal kidney tissue.
Correlation between BIRC5 Expression and Outcome of $c c R C C$

The correlation between higher BIRC5 expression and ccRCC progression inspired us to consider whether
Wang/Chen/Dang/Zhang/Wang/Yin/Jia/ Zhang 


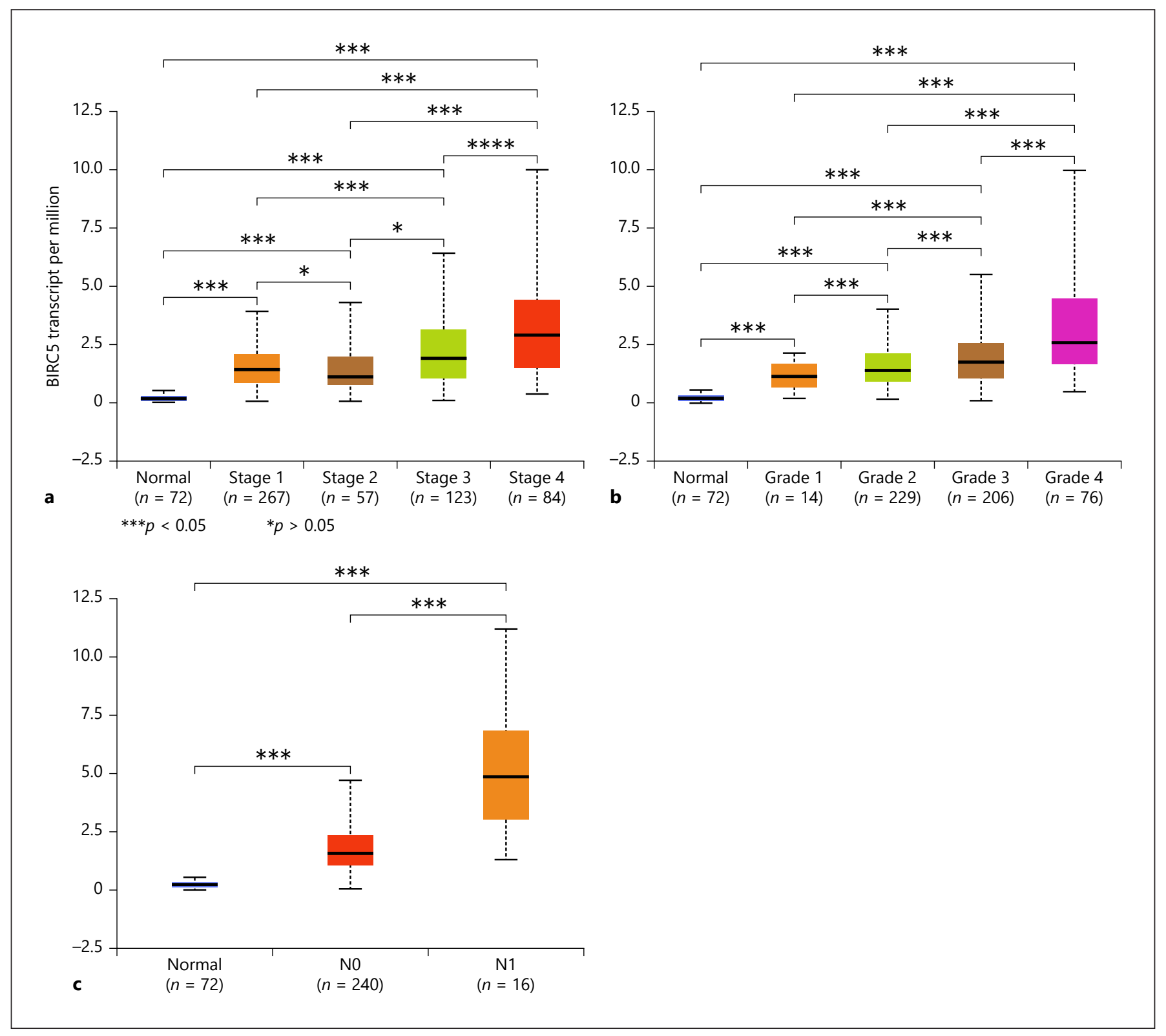

Fig. 2. The relationship between BIRC5 expression of ccRCC and clinical parameters. BIRC5 expression was differential in clinical stage, grade, and nodal metastasis parameters $(p<0.05)$. BIRC5 expression and stage (a); BIRC5 expression and grade (b); and BIRC5 expression and nodal metastasis (c). ccRCC, clear-cell renal cell carcinoma.

Differential Expression of BIRC5 in the Plasma between Early ccRCC and Normal People

We analyzed the BIRC5 expression in paired samples by quantitative reverse transcriptase-polymerase chain reaction. Our data revealed that mRNA expression levels of BIRC5 were significantly higher in ccRCC than normal people $(p<0.05$, Fig. 5$)$.

Early Diagnostic and Prognostic Value of BIRC5 in ccRCC Based on TCGA Data

\section{Discussion}

In this study, we used several web portals to explore the early diagnostic and prognostic value of BIRC5 in ccRCC patients by integrated bioinformatics analysis. Compared with a healthy population, the BIRC5 expression level in patients with early stage ccRCC is significantly higher, which means that BIRC5 may be an early 


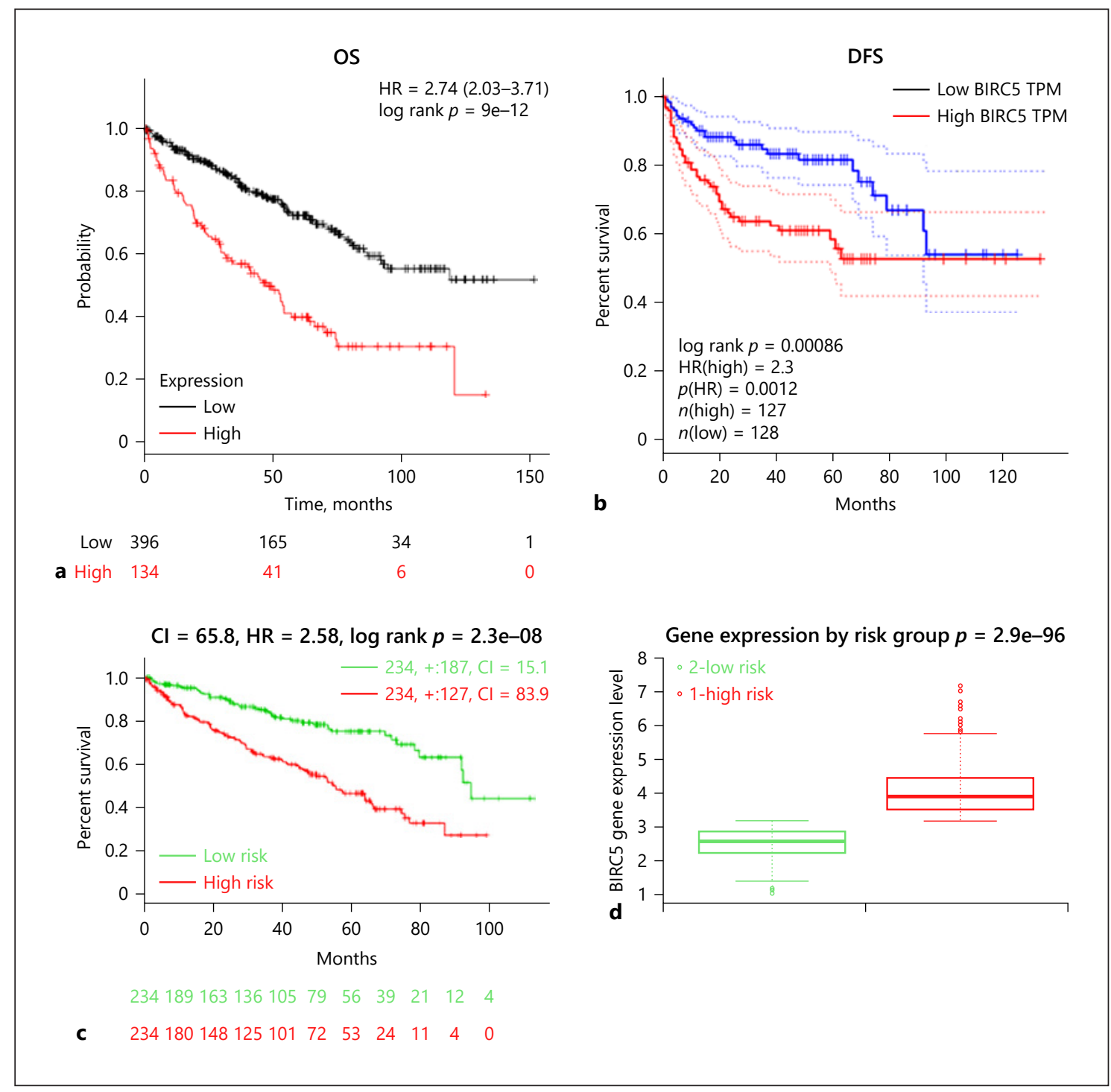

Fig. 3. The relationship between BIRC5 expression and outcome of ccRCC. BIRC5 expression and OS (a); BIRC5 expression and DFS (b); survival across the high- and low-risk group (the numbers below the horizontal axis represent the number of individuals not presenting the event of the corresponding risk group along time) (c); and BIRC5 expression across the high- and low-risk group (d). CI, concordance index; OS, overall survival; DFS, disease-free survival; ccRCC, clear-cell renal cell carcinoma.

diagnostic biomarker for ccRCC. Moreover, BIRC5 expression is highly correlated with the ccRCC clinical and pathological stage, and BIRC5 expression is significantly increased in the higher clinical and pathological stage. We analyzed the relationship between BIRC5 expression and prognosis of ccRCC, and found patients with a high BIRC5 expression, who had a worse OS or DFS. These results suggested that the high expression of BIRC5 prominently correlated with the prognosis and develop- ment of ccRCC, which means that BIRC5 is an important predictor of ccRCC patient prognosis.

With its invasive and chemoresistant characters, ccRCC can be cured by surgery when diagnosed at an early stage. However, there is no simple and rapid method to early detect ccRCC. For example, as a gold standard for ccRCC diagnosis, renal biopsy has a high sensitivity of diagnosis with a low complication rate of $<5 \%$ [12];however, due to the asymptomatic of ccRCC at an 
Fig. 4. The expression of BIRC5 protein of the normal and ccRCC kidney tissue in HPA (magnification: LP $\times 85$; HP $\times 240$ ). HPA, Human Protein Atlas; ccRCC, clearcell renal cell carcinoma.
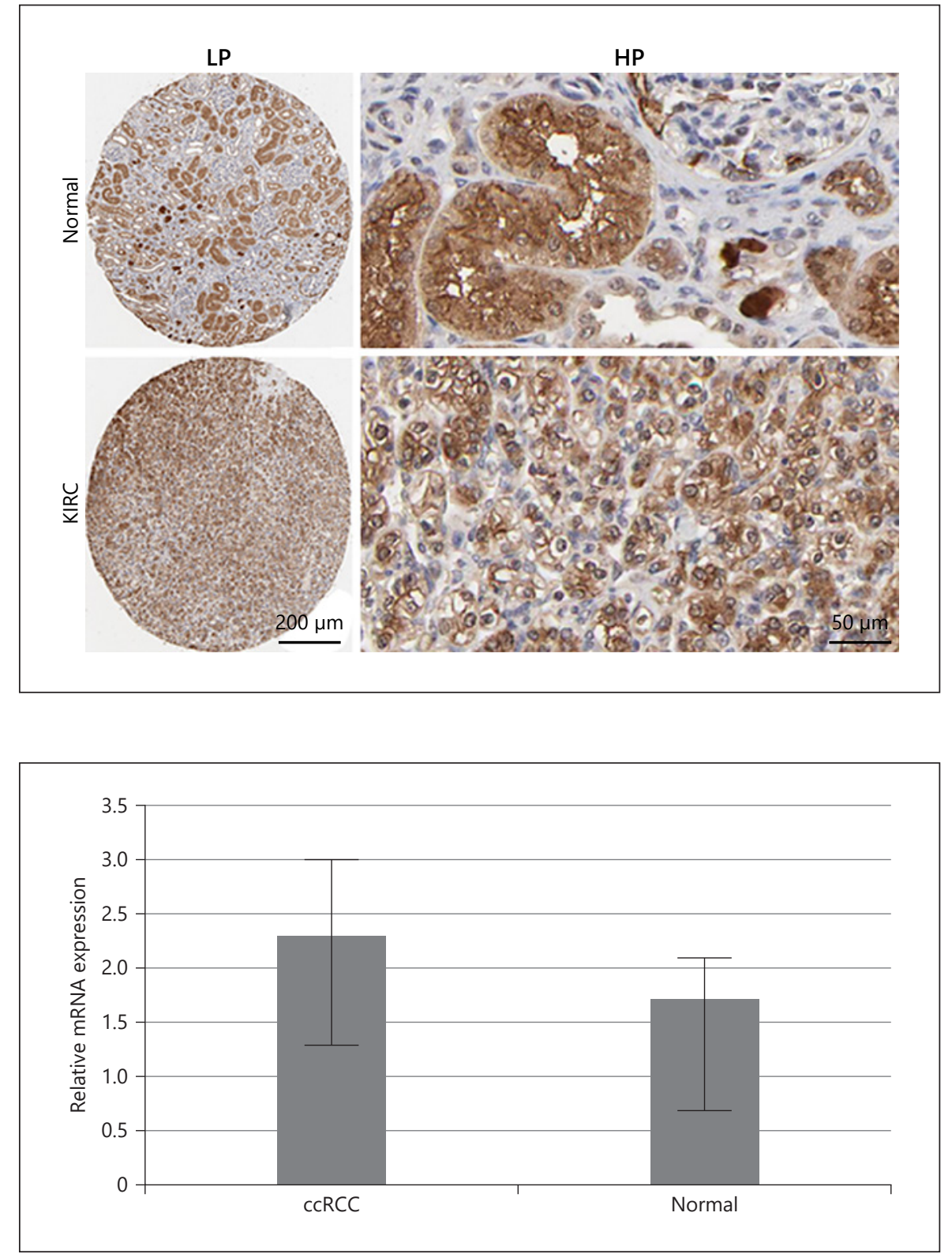

Fig. 5. The expression of BIRC5 in the ccRCC plasma was frequently and significantly higher than normal $(p<0.05)$. Data are represented as mean \pm SEM. ccRCC, clear-cell renal cell carcinoma. makes it a noninvasive method for detecting ccRCC. In our study, the BIRC5 expression level in patients with early stage ccRCC is significantly higher than in a healthy population. So, we measure the expression of BIRC5 in the plasma between early ccRCC and normal people. Although the samples were limited, a significant over expression of BIRC5 was observed by applying Q-PCR in early ccRCC samples compared with the paired normal people. So, we assume survivin may be an early diagnostic biomarker for ccRCC, but it is sensitivity and specificity need to be clear by further clinical trials. 
Previous studies have suggested that increased BIRC5 expression contributes to the negative outcomes of various cancers, such as breast, lung, colorectal, prostate, and ovarian cancers [7]. Cao et al. [19] found that a high BIRC5 expression is related to tumor progression and a poor prognosis of lung adenocarcinoma. Narimani et al. [20] found that knockdown of BIRC5 induces apoptosis in acute myelocytic leukemia. What's more, 2 meta-analyses suggested that a high BIRC5 expression was associated with a poor prognosis and a more advanced pathological stage of RCC $[21,22]$. In this study, we certify that a high BIRC5 expression is associated with a worse prognosis in ccRCC patients. Our findings are consistent with existing investigations. Therefore, we hypothesis that BIRC5 is a potential biomarker for progression and therapeutic target of ccRCC patients.

BIRC5 is a mitotic spindle checkpoint gene, and is located near the telomeric end of the chromosome 17 [23]. It plays an important role in the regulation of mitosis and apoptosis of the cell, also involved in pathological processes [5]. Its encoded protein, survivin, has 2 phosphorylation sites on its different domains (Thr34 and Thr117) which determines its bifunctional molecule effect on apoptosis and cell proliferation. Thr34 is a site on the regulation of cell apoptosis, when the Thr117 is involved in proliferation and cell cycle [24]. Previous studies demonstrate that BIRC5 is a key target involved in a variety of cancer cell signaling pathways, and the upregulation of BIRC5 may play a role in the following several mechanisms. First, it can antagonize caspase-dependent apoptosis, and activate P53 and its downstream target P21 to achieve the purpose of stalling cell cycle progression [25]. Second, BIRC5 facilitates the invasion and migration of tumor cells mediated through the PI3K/AKT pathway [26] or the TGF- $\beta$ pathway [27]. Another, it can regulate tumor cell proliferation mediated by the $\beta$-catenin pathway [28]. These results may explain BIRC5 was mainly involved in the regulation of cell cycle and apoptosis. Meanwhile, our study indicates that the high expression of BIRC5 promoted the development of ccRCC, which may be through regulating the cell cycle signaling pathway.

The main limitation of our study is that our study was conducted using data from public databases that were not verified in prospective clinical trials. These findings need to be validated in prospective clinical trials. Moreover, as a potential biomarker for early diagnosis, the specific concentration of survivin to diagnose ccRCC needs to be further determined by clinical trials, also its sensitivity and specificity need to be clear. Also, the mechanisms

through which BIRC5 promotes the progression of ccRCC requires further investigation, and the functions of the BIRC5 that impact the development of ccRCC needs to be investigated further through in vivo and in vitro experiments.

In conclusion, for ccRCC, BIRC5 may serve as a promising early diagnostic or prognostic predictor and therapeutic target. However, further investigations are necessary to confirm the findings of our study.

\section{Statement of Ethics}

The study was approved by the Institutional Review Board of The First Affiliated Hospital of Xian JiaoTong University, Xian, China (approval no.: XJTU1AF2021LSK-068). All patients provided written informed consent.

\section{Conflict of Interest Statement}

The authors declare that they have no conflicts of interest.

\section{Funding Sources}

This research received no specific grant from any funding agency in the public, commercial, or not-for-profit sectors.

\section{Author Contributions}

All the authors contributed to the study conception and design. Material preparation, data collection, and analysis were performed by Xin Wang, Jianhao Yin, and Rui Jia. The first draft of the manuscript was written by Jingyuan Wang and Min Chen, and all the authors commented on previous versions of the manuscript. All the authors read and approved the final manuscript.

References

Urol Int 2022;106:344-35 DOI: $10.1159 / 000517310$
Wang/Chen/Dang/Zhang/Wang/Yin/Jia/ Zhang
1 Torre LA, Bray F, Siegel RL, Ferlay J, LortetTieulent J, Jemal A. Global cancer statistics, 2012. CA Cancer J Clin. 2015;65(2):87-108.

2 Brian IR, Steven CC, Bernard E. Renal cell carcinoma. Lancet. 2009;373:1119-32.

3 Hakimi AA, Chen L, Kim PH, Sjoberg D, Glickman L, Walker MR, et al. The impact of metformin use on recurrence and cancer-specific survival in clinically localized high-risk renal cell carcinoma. Can Urol Assoc J. 2013; 7(11-12):E687-91.

4 De P, Otterstatter MC, Semenciw R, Ellison LF, Marrett LD, Dryer D. Trends in incidence, mortality, and survival for kidney cancer in Canada, 1986-2007. Cancer Causes Control. 2014;25(10):1271-81. 
5 Mita AC, Mita MM, Nawrocki ST, Giles FJ. Survivin: key regulator of mitosis and apoptosis and novel target for cancer therapeutics. Clin Cancer Res. 2008;14(16):5000-5.

6 Nachmias B, Ashhab Y, Ben-Yehuda D. The inhibitor of apoptosis protein family (IAPs): an emerging therapeutic target in cancer. Semin Cancer Biol. 2004;14(4):231-43.

7 Duffy MJ, O’Donovan N, Brennan DJ, Gallagher WM, Ryan BM. Survivin: a promising tumor biomarker. Cancer Lett. 2007;249(1): 49-60.

8 Tomczak K, Czerwińska P, Wiznerowicz M. The Cancer Genome Atlas (TCGA): an immeasurable source of knowledge. Contemp Oncol. 2015;19(1A):A68-77.

9 Chandrashekar DS, Bashel B, Balasubramanya SAH, Creighton CJ, Ponce-Rodriguez I, Chakravarthi BVSK, et al. UALCAN: a portal for facilitating tumor subgroup gene expression and survival analyses. Neoplasia. 2017; 19(8):649-58.

10 Tang Z, Li C, Kang B, Gao G, Li C, Zhang Z. GEPIA: a web server for cancer and normal gene expression profiling and interactive analyses. Nucleic Acids Res. 2017;45(W1): W98-102.

11 Aguirre-Gamboa R, Gomez-Rueda H, Martínez-Ledesma E, Martínez-Torteya A, Chacolla-Huaringa R, Rodriguez-Barrientos A, et al. SurvExpress: an online biomarker validation tool and database for cancer gene expression data using survival analysis. PLoS One. 2013;8(9):e74250.

12 Caoili EM, Davenport MS. Role of percutaneous needle biopsy for renal masses. Semin Intervent Radiol. 2014;31(1):20-6.
13 Sadat-Khonsari M, Papayannis M, Schriefer $\mathrm{P}$, Kluth L, Meyer C, Schüttfort V, et al. Worth a second look: outcomes of patients with initial finding of regular renal tissue in CT-guided renal tumor biopsies. World J Urol. 2018; 36(5):789-92.

14 Fawzy A, Gaafar R, Kasem F, Ali SS, Elshafei $\mathrm{M}$, Eldeib M. Importance of serum levels of angiopoietin-2 and survivin biomarkers in non-small cell lung cancer. J Egypt Natl Canc Inst. 2012;24(1):41-5.

15 Guney N, Soydine HO, Derin D, Tas F, Camlica H, Duranyildiz D, et al. Serum and urine survivin levels in breast cancer. Med Oncol. 2006;23(3):335-9.

16 Dobrzycka B, Mackowiak-Matejczyk B, Terlikowska KM, Kulesza-Bronczyk B, Kinalski M, Terlikowski SJ. Serum levels of IL-6, IL-8 and CRP as prognostic factors in epithelial ovarian cancer. Eur Cytokine Netw. 2013; 24(3):106-13.

17 Nigam J, Chandra A, Kazmi HR, Singh A, Gupta V, Parmar D, et al. Expression of serum survivin protein in diagnosis and prognosis of gallbladder cancer: a comparative study. Med Oncol. 2014;31(9):167.

18 Ren YQ, Zhang HY, Su T, Wang XH, Zhang L. Clinical significance of serum survivin in patients with pancreatic ductal adenocarcinoma. Eur Rev Med Pharmacol Sci. 2014;18: 3063-8.

19 Cao Y, Zhu W, Chen W, Wu J, Hou G, Li Y. Prognostic value of BIRC5 in lung adenocarcinoma lacking EGFR, KRAS, and ALK mutations by integrated bioinformatics analysis. Dis Markers. 2019;2019:5451290.

20 Narimani M, Sharifi M, Hakhamaneshi MS, Roshani D, Kazemi M, Hejazi SH, et al. BIRC5 gene disruption via CRISPR/Cas9n platform suppress acute myelocytic leukemia progression. Iran Biomed J. 2019;23(6):369-78.
21 Xiong C, Liu H, Chen Z, Yu Y, Liang C. Prognostic role of survivin in renal cell carcinoma: a system review and meta-analysis. Eur J Intern Med. 2016;33:102-7.

22 Xie Y, Ma X, Gu L, Li H, Chen L, Li X, et al. Prognostic and clinicopathological significance of survivin expression in renal cell carcinoma: a systematic review and meta-analysis. Sci Rep. 2016;6:29794.

23 Le Large TY, Mato Prado M, Krell J, Bijlsma MF, Meijer LL, Kazemier G, et al. Bioinformatic analysis reveals pancreatic cancer molecular subtypes specific to the tumor and the microenvironment. Expert Rev Mol Diagn. 2016;16(7):733-6.

24 Wang L, Kang Y, Zheng W, Li L, Shi L, Ma X. Effect on apoptosis and cell cycle of recombinant double negative dominant mutation Survivin (T34/117A) in breast cancer cell B-Cap-37. Biomed Pharmacother. 2014;68(3):277-84.

25 Rauch A, Carlstedt A, Emmerich C, Mustafa A-HM, Göder A, Knauer SK, et al. Survivin antagonizes chemotherapy-induced cell death of colorectal cancer cells. Oncotarget. 2018;9(45):27835-50.

26 Shang X, Liu G, Zhang Y, Tang P, Zhang H, Jiang H, et al. Downregulation of BIRC5 inhibits the migration and invasion of esophageal cancer cells by interacting with the PI3K/ Akt signaling pathway. Oncol Lett. 2018; 16(3):3373-9.

27 Wang B, Li X, Zhao G, Yan H, Dong P, Watari $\mathrm{H}$, et al. miR-203 inhibits ovarian tumor metastasis by targeting BIRC5 and attenuating the TGF $\beta$ pathway. J Exp Clin Cancer Res. 2018;37(1):235.

28 Yang CT, Li JM, Li LF, Ko YS, Chen JT. Stomatin-like protein 2 regulates survivin expression in non-small cell lung cancer cells through $\beta$-catenin signaling pathway. Cell Death Dis. 2018;9(4):425. 